\title{
Chemotherapy increases caspase-cleaved cytokeratin 18 in the serum of breast cancer patients
}

\author{
Engin Ulukaya ${ }^{1}$, Esra Karaagac ${ }^{1}$, Ferda Ari ${ }^{2}$, Arzu Y. Oral ${ }^{1}$, Saduman B. Adim³ ${ }^{3}$, \\ Asuman H. Tokullugil ${ }^{1}$, Türkkan Evrensel ${ }^{4}$ \\ ${ }^{1}$ Medical School of Uludag University, Clinical Biochemistry Department, Bursa, Turkey \\ ${ }^{2}$ Science and Art Faculty of Uludag University, Biology Department, Bursa, Turkey \\ ${ }^{3}$ Medical School of Uludag University, Pathology Department, Bursa, Turkey \\ ${ }^{4}$ Medical School of Uludag University, Medical Oncology Department, Bursa, Turkey
}

Received 5 January 2011

Accepted 25 January 2011

Correspondence to: Prof. Dr. Engin Ulukaya, Medical School of Uludag University, Department of Medical Biochemistry 16059 Gorukle, Bursa, Turkey. Phone: +90 (0)224 29539 13; Fax: +90 (0)224 44282 45; E-mail: eulukaya@uludag.edu.tr

Disclosure: No potential conflicts of interest were disclosed.

Background. Apoptosis is thought to be induced by chemotherapy in cancer patients. Therefore, the measurement of its amplitude may be a useful tool to predict the effectiveness of cancer treatment sooner than conventional methods do.

Patients and methods. In the study presented, apoptosis was assessed with an ELISA-based assay in which caspase-cleaved cytokeratin 18 (M30-antigen), a novel specific biomarker of apoptosis, is measured. Thirty seven patients with malignant (nonmetastatic and metastatic) breast cancer, 35 patients with benign breast disease, and 34 healthy subjects were studied. Cancer patients received neoadjuvant chemotherapy consisting of either fluorouracil, epirubicin, and cyclophosphamide (FEC) or epirubicin plus docetaxel (ED). Apoptosis was detected before chemotherapy, 24 and $48 \mathrm{~h}$ after chemotherapy in the malignant group.

Results. It was found that the baseline apoptosis level in either malignant but nonmetastatic group or benign group was not statistically different from that in the control group ( $p>0.05)$. However, it was statistically significantly higher in the metastatic group than that in the control group $(p<0.05)$. Following the drug application, M30-antigen levels significantly increased at $24 \mathrm{~h}(\mathrm{p}<0.05)$. The baseline M30-antigen levels increased about 3-times in patients showing tumor regression.

Conclusions. M30-antigen level is increased after chemotherapy and its measurement may help clinicians to predict the effectiveness of chemotherapy sooner in breast cancer cases although confirmative larger trials are needed.

Key words: apoptosis; chemotherapy; M30; response to chemotherapy; breast cancer

\section{Introduction}

Breast cancer is the leading cancer type which accounts for the highest mortality rate among woman cancers. ${ }^{1-3}$ Although new chemotherapeutic agents have been introduced into the market, the patient outcome is still not satisfactory. ${ }^{3}$ The improvement of the outcome may be achieved by an early prediction of the response to chemotherapy. For this aim, new biomarker(s), which provide information of the effectiveness of chemotherapy, are required. ${ }^{4}$
Apoptosis-related biomarkers may be of importance in this regard.

The mechanism by which chemotherapy kills the cancer cells is mainly the induction of the apoptotic pathway. ${ }^{5}$ Because the effects of anti-cancer drugs is based on the induction of apoptosis, in vitro evaluation of apoptosis has been used for testing the efficacy of anti-cancer agents. ${ }^{6-8}$ If apoptosis in serum can be measured by a biomarker which results from its induction, this may be of great importance for the clinicians to predict the response to 
TABLE 1. The characteristics of participants

\begin{tabular}{lc}
\hline Characteristics & $\mathbf{n}$ \\
\hline Control & $\mathbf{3 4}$ \\
$\quad$ Mean age \pm S.D. $(47.2 \pm 10.6)$ & \\
Malignant group & $\mathbf{3 7}$ \\
$\quad$ Mean age \pm S.D. $(51.1 \pm 12)$ & \\
$\quad$ Invasive ductal carcinoma & 29 \\
$\quad$ Invasive lobuler carcinoma & 3 \\
$\quad$ Metastatic breast cancer & 5 \\
Benign group & $\mathbf{3 5}$ \\
$\quad$ Mean age \pm S.D. (40.6 \pm 8.2$)$ & \\
$\quad$ Fibrocystic & 31 \\
$\quad$ Fibroadenoma & 4 \\
Sex & All women \\
Stage & \\
I & 4 \\
II & 17 \\
III & 11 \\
IV & 5 \\
ER (+) (-) & 14 \\
PR (+) & 8 \\
PR (-) & 18 \\
\hline
\end{tabular}

ER, estrogen receptor status; PR, progesterone receptor status

chemotherapy they apply to their patients. It seems that there is such a biomarker which is found in the cytoskeleton.

Cytokeratin 18 (CK 18) is a member of cytoskeletal protein family which is present in epithelial cells. ${ }^{9}$ When apoptosis is induced, CK 18 is cleaved from aspartate amino acids localized at position 238 and 396. Monoclonal antibody M30 recognizes the neoepitope of CK 18 formed after cleavage by the caspases. This newly-formed neoepitope can be regarded as a selective biomarker of apoptosis. ${ }^{10,11}$ In fact, it was reported that the M30-antigen assay, which detects this neoepitope, reflects apoptosis accurately. ${ }^{12}$ It is also reported that M30-antigen is used as a marker for pharmocodynamic studies in cancer. ${ }^{13}$ Because deregulated apoptosis is a common feature of malignancies ${ }^{14}$, its assessment via circulating apoptotic markers have recently been made in some tumor types, such as gastrointestinal cancers. ${ }^{15}$ Recently, it was reported that serum M30-antigen levels may also be a prognostic marker in some tumor types. ${ }^{16,17}$ In another study, M30antigen was reported to be associated with the survival in advanced gastric carcinoma patients. ${ }^{18}$

In addition to its being used as a prognostic marker in tumors, M30-antigen may provide important information regarding the response to therapy. Thus, it may be useful for the estimation of the efficacy of therapy. ${ }^{19}$ Kramer et al. presented that serum M30-antigen levels increased after docetaxel regimen in prostate cancer. ${ }^{20}$ Similarly, it was demonstrated that M30-antigen levels increased after chemotherapy in testicular cancer patients. ${ }^{16}$ Our group previously showed that M30-antigen levels increased as a response to therapy in breast cancer patients but we did not measure its levels in benign breast diseases and healthy subjects. ${ }^{21}$

Therefore, we investigated if M30-antigen is increased in breast cancer patients as well as in benign breast diseases in comparison with healthy subjects. We also measured its level after the application of chemotherapy in neoadjuvant setting. We found that chemotherapy leads to a significant increase in M30-antigen levels in serum of breast cancer patients. Thus, it may be used as a biomarker for the prediction of response to chemotherapy in breast cancer patients.

\section{Patients and methods}

\section{Patient selection, treatment and assessment of clinical tumor response}

The characteristics of the study participants are presented in Table 1. Patients with previously untreated, histological confirmed invasive breast cancer were eligible. The patient selection and eligibility criteria to be enrolled to the study were made according to the previous study performed by our group. ${ }^{21}$ Briefly, their performance status $\leq 2$ by ECOG. Core needle biopsy was used for the histological confirmation of the tumor. The patients were treated with FEC or ED regimens: FEC regimen consisted of 5-fluorouracil (EBEWE Pharma, Austria) $500 \mathrm{mg} / \mathrm{m}^{2}$, epirubicin (EBEWE Pharma, Austria) $100 \mathrm{mg} / \mathrm{m}^{2}$, cyclophosphamide (BAXTER, Germany) $500 \mathrm{mg} / \mathrm{m}^{2}$ while ED regimen consisted of epirubicin $75 \mathrm{mg} / \mathrm{m}^{2}$ and docetaxel (EBEWE Pharma, Austria) $80 \mathrm{mg} / \mathrm{m}^{2}$. All drugs were administered on day 1 , at every 21 days.

The response to treatment was assessed after the completion of four cycles of neoadjuvant chemotherapy by standard breast calipers and graded into: (a) clinical complete response (no tumor measurable); (b) clinical partial response ( $\geq 50 \%$ reduction in tumor size); (c) clinical stable disease $(<50 \%$ reduction or an increase in tumor size of $\leq 50 \%$ ); and (d) clinical progressive disease ( $>50 \%$ increase in tumor size or suspicious new lesion). According to the classification above, the complete and partial responses were defined as a regressive group. The other two groups were the stable group showing stable disease and the 
TABLE 2. M30-antigen levels in different groups

\begin{tabular}{lcccc}
\hline M30-antigen & $\begin{array}{c}\text { Control group } \\
(\mathbf{n}=\mathbf{3 4})\end{array}$ & $\begin{array}{c}\text { Benign group } \\
(\mathbf{n}=\mathbf{3 5})\end{array}$ & $\begin{array}{c}\text { Nonmetastatic group } \\
(\mathbf{n}=\mathbf{3 2})\end{array}$ & $\begin{array}{c}\text { Metastatic group } \\
(\mathbf{n}=\mathbf{5})\end{array}$ \\
\hline Mean \pm S.D. & $127 \pm 46$ & $173 \pm 224$ & $182 \pm 336$ & $333 \pm 184$ \\
(min-max) & $71-340$ & $68-1295$ & $58-2010$ & $159-607$ \\
Median & 114 & 107 & 118 & 350 \\
p-Value & & $>0.05^{*}$ & $>0.05^{* *}$ & $<0.05^{* * *}$ \\
\hline
\end{tabular}

*Comparison of control group and benign group, Mann-Whitney U test

** Comparison of control group and nonmetastatic group, Mann-Whitney U test

***C Comparison of control group and metastatic group, Mann-Whitney U test

progressive group showing progression as given above. The informed consent was obtained from the participants and the local ethic committee approved the study.

\section{M30-antigen detection}

The serum samples of malignant cases were collected prior to chemotherapy (baseline M30antigen level), and 24 and $48 \mathrm{~h}$ after the treatment. Therefore, the acute (short term) effect of the therapy was actually assessed ignoring the long term effects. The sera of benign and healthy control subjects were collected at the time of admission only. The sera were stored at $-80^{\circ} \mathrm{C}$ until the assessment. An ELISA assay (a solid phase, two-site immunosorbent assay) was used to measure M30-antigen by using a commercial kit (M30-Apoptosense ELISA kit, Peviva, Sweden). Measurement was performed according to the instructions of the manufacturer. The absorbance was finally measured in a microplate reader (FlashScan, Jena, Germany) at $450 \mathrm{~nm}$ and the M30-antigen levels were estimated by the standard curve. The concentration of the M30-antigen was expressed as unit per liter (U/L).

\section{Histopathological evaluation}

Tissue specimens were fixed in 10\% buffered formalin ( $\mathrm{pH} 7.4$ ) and embedded in paraffin. $5 \mu \mathrm{m}$ thick sections were cut and stained with hematoxylin and eosin. Estrogen (ER) and progesterone (PgR) receptor status were assessed by immunohistochemistry. All specimens were examined by an experienced pathologist who was unaware of the clinical data. Only 22 patients' samples were accessible. The proportion of ER and PgR positive cells was determined as the percentage of invasive tumor cells. The threshold of $10 \%$ positivity was chosen as a cut-off value.

\section{Statistical analysis}

The statistical analysis was performed using SPSS 13.0 (SPSS Inc., Chicago, IL, USA). All values are presented as mean ( \pm standard deviation- S.D.) and median. In the case of the distribution of parameters did not show normal distribution, nonparametric statistics (Kruskal-Wallis and Mann Whitney-U tests) were used. Wilcoxon Rank Sum test was also used to compare two dependent samples represented by M30-antigen levels before and after chemotherapy. The relationship between M30-antigen levels and parameters were analyzed by Pearson Correlation. Statistical significance was assigned to $p$-values less than 0.05 .

\section{Results}

\section{Characteristics of the study groups}

The characteristics of participants are given in Table 1. The healthy control group included 34 people, while the malignant (metastatic and nonmetastatic) group and the benign group included 37 and 35 patients, respectively. Among the malignant group, most of them had invasive ductal carcinoma $(n=29)$, while only 5 of them were metastatic breast cancer cases.

\section{Serum M30-antigen levels are elevated in metastatic breast cancer patients}

The baseline level of serum M30-antigen was measured in control, benign and malignant groups. It was found that the mean M30-antigen levels in control, benign, nonmetastatic and metastatic group were $127 \pm 46,173 \pm 224,182 \pm 336$, and 333 \pm 184 , respectively. A statistically significant difference between the groups was observed $(p<0.05$; Kruskal-Wallis and Mann-Whitney U tests) (Table 2 ). The mean of the metastatic malignant group 
TABLE 3. M30-antigen levels after chemotherapy $(n=11)$

\begin{tabular}{lcc}
\hline M30-antigen level (U/L) & $\begin{array}{c}\text { Before chemotherapy (baseline } \\
\text { level) }\end{array}$ & 24 h after chemotherapy \\
\hline Mean \pm S.D & $316 \pm 564$ & $809 \pm 1526$ \\
(min-max) & $96-2010$ & $98-4986$ \\
Median & 136 & 143 \\
P-Value & & $<2-2586$ \\
\hline
\end{tabular}

* Comparison of M30-antigen levels before chemotherapy and those $24 \mathrm{~h}$ after chemotherapy, Wilcoxon Sign Rank test

** Comparison of M30-antigen levels before chemotherapy and those $48 \mathrm{~h}$ after chemotherapy, Wilcoxon Sign Rank test.

TABLE 4. The M30-antigen levels in $\operatorname{ER}(-), E R(+)$ and $\operatorname{PgR}(-), \operatorname{PgR}(+)$ groups

\begin{tabular}{|c|c|c|c|c|}
\hline M30-antigen level (U/L) & $\begin{array}{l}\text { ER (+) } \\
n=14\end{array}$ & $\begin{array}{c}\text { ER }(-) \\
n=8\end{array}$ & $\begin{array}{c}\mathrm{PgR}(+) \\
n=18\end{array}$ & $\underset{n=4}{\operatorname{PgR}(-)}$ \\
\hline Mean $\pm S . D$ & $153 \pm 39$ & $183 \pm 31$ & $148 \pm 30$ & $235 \pm 51$ \\
\hline (min.-max.) & $58-607$ & $86-383$ & $58-607$ & $146-383$ \\
\hline$p$-Value & & & & \\
\hline
\end{tabular}

Only 22 patients' data were obtained for the evaluation of ER and PgR status.

*Comparison of ER(-) group and ER(+) group, Mann-Whitney U test

** Comparison of PgR(-) group and PgR(+) group, Mann-Whitney U test

was significantly higher than either benign or control group $(\mathrm{p}<0.05)$. There was no significant difference between the control group and either the nonmetastatic malignant group or the benign group. The mean M30-antigen level of the metastatic group was significantly higher than that in the nonmetastatic group.

\section{M30-antigen level increases following chemotherapy}

Eleven nonmetastatic breast cancer patients accepted to donate serum sample after chemotherapy. Blood samples were collected prior to chemotherapy and 24 and $48 \mathrm{~h}$ after the application of chemotherapy. The baseline, $24 \mathrm{~h}$ and $48 \mathrm{~h}$ after chemotherapy levels were $316 \pm 564,809 \pm 1526$, and $584 \pm$ 874 , respectively. The baseline M30-antigen levels increased more than 2-fold $24 \mathrm{~h}$ after chemotherapy $(p<0.05)$ (Table 3). In addition, M30-antigen level at $48 \mathrm{~h}$ following chemotherapy was still higher than the baseline level but it was not statistically significant $(\mathrm{p}>0.05)$.

\section{Relationship between M30-antigen level and receptor status, stage, and routine tumor markers}

It has been found that M30-antigen levels differ depending on the ER or PgR status. ER negative (183 $\pm 31)$ or PgR negative $(235 \pm 51)$ cases had higher
M30-antigen levels compared to those in ER (153 \pm $39)$ or PgR $(148 \pm 30)$ positive cases $(\mathrm{p}<0.05)$, respectively (Table 4).

M30-antigen levels differ depending on the stage (Table 5). Stage IV patients seem to have the highest levels. There was no significant difference between stage II and III but stage IV cases had significantly higher levels compared to those in either stage II or III.

The correlation between M30-antigen and the routine clinical chemistry parameters was analyzed. But there was no significant correlation between them (data not shown). In addition, there was no correlation between baseline M30-antigen levels and age $(r=0.045, p=0.647)$. The levels of lactate dehydrogenase (LDH), alkaline phosphatase and platelet count were measured in patients with malignant breast cancer and healthy controls. There was no statistically significant difference between the groups (data not shown).

\section{Relationship between M30-antigen and tumor response to chemotherapy in neoadjuvant setting}

Eleven malignant cases were classified into three groups according to their responses to chemotherapy: regressive group $(n=5)$ consisting of clinical complete or partial responses, stable group $(n=4)$, and progressive group $(n=1)$. One patient's response was not evaluated although post-chem- 
TABLE 5. Comparison of the M30-antigen levels according to the stage of disease

\begin{tabular}{lccc}
\hline M30-antigen level $(\mathbf{U} / \mathrm{L})$ & $\begin{array}{c}\text { Stage II } \\
(\mathbf{n}=17)\end{array}$ & $\begin{array}{c}\text { Stage III } \\
(\mathbf{n}=11)\end{array}$ & $\begin{array}{c}\text { Stage IV } \\
(\mathbf{n}=5)\end{array}$ \\
\hline Mean \pm S.D & $242 \pm 110$ & $117 \pm 15$ & $333 \pm 184$ \\
(min-max) & $72-2010$ & $59-211$ & $159-607$ \\
Median & 131 & 113 & 351 \\
$p$-Value & $p<0.05^{*}$ & $p<0.05^{* *}$ & \\
\hline
\end{tabular}

* Comparison of the M30-antigen levels between stage 2 and 4, Mann-Whitney U test

** Comparison of the M30-antigen levels between stage 3 and 4, Mann-Whitney U test

TABLE 6. Classification into the responses to chemotherapy in the neoadjuvant setting and their M30-antigen values (U/L, $\pm S S)$

\begin{tabular}{llll} 
& Stable group, $(\mathrm{n}=4)$ & Regressive group, $(\mathrm{n}=5)$ & Progressive group, $(\mathrm{n}=1)$ \\
\hline Before Chemotherapy & $137 \pm 46$ & $544 \pm 820$ & 110 \\
24 h after Chemotherapy & $115 \pm 11$ & $1633 \pm 407$ & 143 \\
48 h after Chemotherapy & $116 \pm 23$ & $1076 \pm 1150$ & 502 \\
\hline
\end{tabular}

The statistical evaluation was not performed due to the low number of cases.

otherapy M30-antigen level of that patient was available. As it is shown on Table 6, M30-antigen level of both the stable group and the progressive group did not significantly change after chemotherapy, while it sharply increased in the regressive group $24 \mathrm{~h}$ after chemotherapy. It increased about 3-fold (from 544 to $1633 \mathrm{U} / \mathrm{L}$ ) in this group, implying the apoptosis-inducing effect of drugs applied. However, the differences were not statistically evaluated due to a low number of cases.

\section{Discussion}

In the study presented, we measured the M30antigen levels before and after chemotherapy to investigate its relation with response to treatment. We found that M30-antigen significantly increased following chemotherapy. This may give an idea of the effectiveness of chemotherapy applied in neoadjuvant setting. Neoadjuvant chemotherapy is increasingly being applied in the management of patients with large $(\geq 3 \mathrm{~cm})$ and locally advanced breast cancer. Although neoadjuvant chemotherapy may lead to similar disease-free and overall survival rates with those obtained with adjuvant chemotherapy, the response of breast cancer patients to neoadjuvant chemotherapy was found as the most important predictive factor for the survival. ${ }^{22-24}$ However, which patient would response to the neoadjuvant chemotherapy is still unpredictable. That is why we believe that the measurement of apoptosis, which is induced by anticancer drugs, may be of great importance in the prediction of response to therapy. This may be achieved by measuring the M30-antigen levels in serum and the clinicians may thus predict better the outcome of their patients by using this tool.

Death of tumor cells generates detectable protein products in the patient's circulation, which may be used for cancer diagnostics and/or monitoring of therapy efficacy. ${ }^{25}$ Apoptosis is a form of regulated cell death that is characterized by specific structural changes, mediated by proteases of the caspase family. ${ }^{26}$ Caspase activity itself or the presence of specific degradation products can be used for the detection of tumor cell apoptosis. The M30 antibody detects a caspase-degraded product, CK18-Asp396 (also called M30-antigen), of the important cytoskeletal protein called cytokeratin 18 of epithelial cells. Cytokeratin 18 is expressed by most carcinomas, including those of breast, prostate, lung and colon. ${ }^{11}$

Treatment-induced changes in growth dynamics (apoptosis and proliferation) in breast cancer are essential to determine the response or resistance of tumors to chemotherapy. The early detection of chemosensitive tumor with the assessment of apoptosis or different techniques may facilitate the individualized-chemotherapy. It has previously been shown that circulating M30-antigen levels increased in patients with various cancer types and, furthermore, it increased during chemotherapy. ${ }^{21,27,28}$ For instance, the docetaxel treatment increased levels of M30-antigen in the serum of breast cancer patients, indicating apoptotic 
death of tumor cells, while the cyclophosphamide/ epirubicin/5-fluorouracil treatment led to a heterogenous response with regard to cell death mode. ${ }^{29}$ Our group previously reported that M30-antigen increased 4-fold after chemotherapy in lung cancer patients. ${ }^{30}$ In accordance with this finding, in this study presented, we observed that M30-antigen level was significantly increased 24 and $48 \mathrm{~h}$ after the chemotherapy in breast cancer patients. In fact, preclinical and clinical studies have shown that apoptosis significantly increases 1 to 3 days after chemotherapy administration. ${ }^{31-33}$

In the present study, we found that there was no statistically significant difference between the nonmetastatic and the control group in terms of M30antigen levels ( $p>0.05)$. In supporting this finding, there was no statistically significant difference between the malignant group (202 \pm 84$)$ and the control group (187 \pm 58$)$ in terms of baseline LDH level, which also represents cell death in serum. However, this may depend on the type of tumor. In the patients with disseminated testicular germ cell tumor, circulating M30-antigen levels were found to be correlated with classic prognostic markers including LDH probably reflecting tumor load. ${ }^{16}$

In contrast to inexistence of M30-antigen increase in the non-metastatic group compared to the control group, M30-antigen level was significantly higher in the metastatic group than that in the control group $(\mathrm{p}<0.05)$. This implies that the aggressiveness (metastatic ability) of tumor mass may have an impact on the serum level of M30antigen. This increase may also be explained by the differentiation level of the tumor cells. It is highly possible that the stage or the total size of the tumor mass seems to affect the M30-antigen levels in serum. In fact, in the Olofsson's study, there was a clear relationship between the tumor size and the M30-antigen levels. ${ }^{29}$ In this study, stage IV patients had much higher M30 antigen levels than those either stage II or III patients. Thereby, there must be a close link between apoptosis and both malignancy itself and the extension of malignancy. In agreement with this, we did not find any statistically significant increase in M30antigen levels in the benign group, compared to the control group.

Several studies demonstrated that M30-antigen levels were higher in ER negative breast tumors than ER-positive tumors ${ }^{21,28}$, consistent with our results. Furthermore, we also found that M30-antigen levels were higher in PgR negative tumors, compared to PgR-positive ones. However, this needs to be confirmed by larger clinical studies. In fact, the weakness of our study was the low number of patients studied although the results were interesting.

\section{Conclusions}

These findings indicate that serum M30-antigen is increased following FEC-based or ED-based chemotherapy. Thus, the measurement of its serum level may be a useful tool to predict the effectiveness of chemotherapy sooner in breast cancer patients. However, larger clinical studies are required to use it in the clinics routinely.

\section{Acknowledgement}

We thank to Uludag University Research Fund for providing us with the kits.

\section{References}

1. Benson JR, Jatoi I, Keisch M, Esteva FJ, Makris A, Jordan VC. Early breast cancer. Lancet 2009; 373: 1463-79.

2. Plesnicar A, Golicnik M, Fazarinc IK, Kralj B, Kovac V, Plesnicar BK. Attitudes of midwifery students towards teaching breast-self examination. Radiol Oncol 2010; 44: 52-6.

3. Ovcaricek T. Frkovic SG, Matos E, Mozina B, Borstnar S. Triple negative breast cancer - prognostic factors and survival. Radiol Oncol 2011; 45: 46-52.

4. Strojan P. Cysteine cathepsins and stefins in head and neck cancer: an update of clinical studies. Radiol Oncol 2008; 42: 69-81.

5. Hickman JA, Beere HM, Wood AC, Waters CM, Parmar R. Mechanisms of cytotoxicity caused by antitumour drugs. Toxicol Lett 1992; 64: 553-61.

6. Ohmori T, Podack ER, Nishio K, Takahashi M, Miyahara Y, Takeda Y, et al. Apoptosis of lung cancer cells caused by some anti-cancer agents (MMC, CPT-11, ADM) is inhibited by bcl-2. Biochem Biophys Res Commun 1993; 192: 30-6.

7. Walton MI, Whysong D, O'Connor PM, Hockenbery D, Korsmeyer SJ, Kohn KW. Constitutive expression of human $\mathrm{Bcl}-2$ modulates nitrogen mustard and camptothecin induced apoptosis. Cancer Res 1993; 53: 1853-61.

8. Hagg M, Bivén $\mathrm{K}$, Ueno T, Rydlander L, Björklund P, Wiman KG, et al. A nove high-through-put assay for screening of pro-apoptotic drugs. Invest New Drugs 2002; 20: 253-9.

9. Linder S. Cytokeratin markers come of age. Tumour Biol 2007; 28: 189-95.

10. Ueno $\mathrm{T}$, Toi $\mathrm{M}$, Linder S. Detection of epithelial cell death in the body by cytokeratin 18 measurement. Biomed Pharmacother 2005; 59 (Suppl): S359-62.

11. Leers MP, Kölgen W, Björklund V, Bergman T, Tribbick G, Persson B, et al. Immunocytochemical detection and mapping of a cytokeratin 18 neoepitope exposed during early apoptosis. J Pathol 1999; 187: 567-72.

12. Zhang L, Kavanagh BD, Thorburn AM, Camidge DR. Preclinical and clinical estimates of the basal apoptotic rate of a cancer predict the amount of apoptosis induced by subsequent proapoptotic stimuli. Clin Cancer Res 2010; 16: 4478-89.

13. Linder S, Olofsson MH, Herrmann R, Ulukaya E. Utilization of cytokeratinbased biomarkers for pharmacodynamic studies. Expert Rev Mol Diagn 2010; 10: 353-9.

14. Holdenrieder S, Stieber P. Circulating apoptotic markers in the management of non-small cell lung cancer. Cancer Biomarkers 2010; 6: 197-210. 
15. Brandt D, Volkmann X, Anstätt $M$, Länger F, Manns MP, Schulze-Osthoff $K$, et al. Serum biomarkers of cell death for monitoring therapy response of gastrointestinal carcinomas. Eur J Cancer 2010; 46: 1464-73.

16. de Haas EC, di Pietro A, Simpson KL, Meijer C, Suurmeijer AJ, Lancashire $\sqcup$, et al. Clinical evaluation of M30 and M65 ELISA cell death assays as circulating biomarkers in a drug-sensitive tumor, testicular cancer. Neoplasia 2008; 10: $1041-8$.

17. Wu YX, Wang JH, Wang $\mathrm{H}$, Yang $\mathrm{XY}$. Study on expression of Ki-67, early apoptotic protein M30 in endometrial carcinoma and their correlation with prognosis. Zhonghua Bing Li Xue Za Zhi 2003; 32: 314-8.

18. Yaman E, Coskun U, Sancak B, Buyukberber S, Ozturk B, Benekli M. Serum M30 levels are associated with survival in advanced gastric carcinoma patients. Int Immunopharmacol 2010; 10: 719-22.

19. Beachy SH, Repasky EA. Using extracellular biomarkers for monitoring efficacy of therapeutics in cancer patients: an update. Cancer Immunol Immunother 2008; 57: 759-75.

20. Kramer G, Erdal H, Mertens HJ, Nap M, Mauermann J, Steiner G, et al. Differentiation between cell death modes using measurements of different soluble forms of extracellular cytokeratin 18. Cancer Res 2004; 64: 1751-6.

21. Demiray M, Ulukaya EE, Arslan M, Gokgoz S, Saraydaroglu O, Ercan I, et al. Response to neoadjuvant chemotherapy in breast cancer could be predictable by measuring a novel serum apoptosis product, caspase-cleaved cytokeratin 18: a prospective pilot study. Cancer Invest 2006; 24: 669-76.

22. Fisher B, Bryant J, Wolmark N, Mamounas E, Brown A, Fisher ER, et al. Effect of preoperative chemotherapy on the outcome of women with operable breast cancer. J Clin Oncol 1998; 16: 2672-85.

23. Bonadonna G, Valagussa P, Brambilla C, Ferrari L, Moliterni A, Terenziani M et al. Primary chemotherapy in operable breast cancer: eight-year experience at the Milan Cancer Institute. J Clin Oncol 1998; 16: 93-100.

24. Scholl SM, Beuzeboc P, Harris AL, Pierga JY, Asselain B, Palangié T, et al. Is primary chemotherapy useful for all patients with primary invasive breast cancer? Recent results. Cancer Res 1998; 152: 217-26.

25. Holdenrieder S,Stieber P. Apoptotic markers in cancer. Clin Biochem 2004 37: 605-617.

26. Degterev A, Yuan J. Expansion and evolution of cell death programmes. Nat Rev Mol Cell Biol 2008; 9: 378-90

27. Ozturk B, Coskun U, Sancak B, Yaman E, Buyukberber S, Benekli M. Elevated serum levels of M30 and M65 in patients with locally advanced head and neck tumors. Int Immunopharmacol 2009; 9: 645-8.

28. Ueno T, Toi M, Bivén $\mathrm{K}$, Bando $\mathrm{H}$, Ogawa $\mathrm{T}$, Linder $\mathrm{S}$. Measurement of an apoptotic product in the sera of breast cancer patients. Eur J Cancer 2003; 39: $769-74$

29. Olofsson MH, Ueno T, Pan Y, Xu R, Cai F, van der Kuip H, et al. Cytokeratin-18 is a useful serum biomarker for early determination of response of breast carcinomas to chemotherapy. Clin Cancer Res 2007; 13: 3198-206.

30. Ulukaya E, Yilmaztepe A, Akgoz S, Linder S, Karadag M. The levels of caspase cleaved cytokeratin 18 are elevated in serum from patients with lung cancer and helpful to predict the survival. Lung Cancer 2007; 56: 399-404.

31. Meyn RE, Stephens LC, Hunter NR, Milas L. Apoptosis in murine tumors treated with chemotherapy agents. Anticancer Drugs 1995; 6: 443-50.

32. Ellis PA, Smith IE, McCarthy K, Detre S, Salter J, Dowsett M. Preoperative chemotherapy induces apoptosis in early breast cancer. Lancet 1997; 349: 849.

33. Green AM, Steinmetz ND. Monitoring apoptosis in real time. Cancer $J 2002$ 8. 82-92. 